$96.92 \mathrm{~kg}$ for OGD (21.85 g per OGD); $35.30 \mathrm{~kg}$ for sigmoidoscopy (15.68 g per sigmoidoscopy); and $159.38 \mathrm{~kg}$ for colonoscopy (50.84 g per colonoscopy). In 1 year, the total plastic generated from sterile water bottles alone for these procedures was $291.6 \mathrm{~kg}$. The total cost for sterile water was $£ 3588.80$ ( $£ 1192.80$ for OGD, $£ 434.40$ for sigmoidoscopy and $£ 1961.60$ for colonoscopy).

Conclusion We conclude that significantly large volumes of sterile water and single use plastic are being used in endoscopy. This practice is not only costly but also has a detrimental impact on the environment due to the large amount of plastic generated. Reduction of single use plastic needs to be a priority for a greener and more sustainable practice of endoscopy.

\section{P360 PILOT INTRODUCTION OF A TRIAGE SYSTEM REDUCES THE WORKLOAD OF THE HPB MDT}

Claire Grant*, Edward Alababra, Dawn Drury, Yutaro Higashi, Christopher Clarke, Uday Bannur, Philip Kaye, Asma Haider, Arvind Arora, Ankit Rao, Aloysius Aravinthan, Suresh Venkatachalapathy. Nottingham University Hospitals NHS Trust, Nottingham, UK

\subsection{6/gutjnl-2020-bsgcampus.434}

Introduction The East Midlands Hepatobiliary Multidisciplinary Team (HPB MDT) serves a population of 2.4 million people. The high volume of referrals and increasing complexity of cases has consistently led to significant time constraint for case discussion and potential delay in timely delivery of care. Therefore, a triage system was introduced to identify cases where a full discussion was unnecessary or premature. The aim was to improve the operation of MDT meetings (MDTM) and deliver a streamlined, transparent pathway for all patients.

Methods A triage system was piloted in two of four regional trusts from January 2019. The triage team included two hepatologists, a HPB surgeon, a specialist nurse and an administrator. In line with national guidelines and local policies, triage pathways were developed for cases for which discussion in the main MDTM would not result in additional benefit. These include (1) registration only (2) probable benign pancreatic/liver lesions under surveillance (3) HCC on established treatment (4) advanced HPB cancers on established chemotherapy (5) patients with inadequate investigations (6) post-operative histology. Pathways were reviewed and approved at relevant governance meetings. All referrals were reviewed by the triage team 48 hours prior to the MDTM and two lists were created; one for triage and one for full discussion. Outcomes for those on the triage list were given after review and approval from the radiologist and/or histopathologist. For the first 4 weeks of implementation, all triage decisions were also reviewed at the MDTM. Data on the triage process were prospectively collected from January to December 2019. These were compared with the number of patients who were discussed in the previous 4 years. All statistical analyses were performed using GraphPad Prism 8 (San Diego, CA).

Results A total 2,686 referrals were received in 2019, which was comparable to the previous 4 years $(p=0.11$, figure 1$)$. Adoption of the triage system led to a mean reduction of 37 patients/month to the MDTM $(\mathrm{p}<0.0001$, figure 1$)$. This was a $15 \%$ reduction in workload for the overall MDT and a $25 \%$ reduction for the 2 trusts that participated. All triaged patients were given a recommendation for further management within the same time period for full MDT discussion. There were no reports of harm or complaints attributable to the triage system during this period.

Conclusions The above results confirm that streamlining of the HPB MDT pathway is feasible and significantly reduces the numbers discussed in the full MDTM without compromising patient care.

\section{P361 THE RELIABILITY OF 'COFFEE-GROUND' VOMITING AS AN INDICATOR OF ACTIVE UGIB: A TERTIARY CENTRE EXPERIENCE}

Rumneek Hampal*, Dean-Martin Borrow, Ikram Nasr, Shuvra Ray. Department of Gastroenterology, Guy's and St. Thomas' NHS Foundation Trust, London, UK

\subsection{6/gutjnl-2020-bsgcampus.435}

Introduction Acute Upper GI Bleeds (UGIB) are a common medical emergency and account for significant morbidity and mortality. 'Coffee-ground' vomiting is considered indicative of UGIB however its reliability as an indicator of active bleeding is contentious. OGD is the current gold standard in the diagnosis and management of UGIB however UGI capsule endoscopy is an emerging alternative which can rapidly screen for bleeding and has benefits over OGD in terms of invasiveness, patient tolerance, rapid deployment anywhere and thus reduced hospital admission rates and ultimately cost-effectiveness. The aim of this study was to establish whether coffee-ground vomiting is a reliable indicator of active UGIB.

Methods Electronic referrals for OGD, which contained the phrase 'coffee-ground vomiting', were analysed and endoscopy reports assessed to identify whether active UGIB was identified.

Results During a 12-month period; 552 electronic endoscopy referrals for UGIB were submitted. 62 referrals contained the specific phrase 'coffee-ground vomiting'; 9 patients were excluded. 33/53 referrals came from inpatient medical wards. 35 patients were male, mean age at time of referral was 67.5 years $(22.0$ - 90.0). Mean Glasgow Blatchford Score at referral was $7.4(1.0$ - 15.0). Mean time between referral and endoscopy was 1.3 days $(0.0-12.0)$.

The most common endoscopic diagnoses were oesophagitis (16/53 cases) and gastritis (9/53 cases). OGD findings were normal in 10 cases. Active bleeding was identified in 6 patients and in 2 patients a visible vessel was visualised without evidence of active bleeding at endoscopy. 4 patients required endotherapy namely injection and clipping, 1 patient required variceal banding. All 6 patients with confirmed active bleeding on endoscopy had melaena on presentation.

Mean length of stay (LOS) was 8 days $(1.0$ - 203.0) and in 1 case a repeat OGD was required during the same admission.

Conclusions The results of this study demonstrate that only a minority of OGDs performed for the indication of 'coffeeground' vomiting demonstrated active UGIB. In addition, a significant proportion of the cohort had a normal study. No patients with coffee ground vomiting without melaena had active UGIB and none of these patients required endotherapy.

This study could be used to support the proposal that patients with coffee-ground vomitus without other 